\title{
Regular Orientations, Arboricity, and Augmentation *
}

\author{
Hubert de Fraysseix and Patrice Ossona de Mendez \\ CNRS UMR 0017, EHESS, 54 Boulevard Raspail, 75006, Paris, France
}

\section{Introduction}

Regular orientations, that is orientations such that almost all the vertices have the same indegree, relates many combinatorial and topological properties, such as arboricity, page number, and planarity. Those orientations are a basic tool in solving combinatorial problems that preserve topological properties. Planar augmentations are a simple example of such problems.

Augmentation problems are concerned with the addition of dummy edges to a graph in order to get some connectivity or maximality properties. For instance, the problem of finding the minimum number of edges to augment a graph to a biconnected graph has been solved in [2]. If the original graph is planar and if it is required to preserve the planarity, the problem is NP-complete [3]. Triangulating a biconnected graph while minimizing the maximum degree has also been proved to be an NP-complete problem.

Regular orientations are also related to arboricity. Those of planar graphs provide very simple algorithms to construct tree decompositions.

We first study constrained orientations of graphs. Then we present a few results on augmentation problems and tree decompositions.

\section{Existence of regular orientations}

The necessary and sufficient conditions for a regular orientation to exist is a consequence of the following simple lemma :

Lemma 1. Let $G$ be a graph and $\lambda: V(G) \rightarrow \mathbb{N}$ be a mapping from the vertex set of $G$ to the integer set. Then, there exists an orientation of $G$ such that each vertex $x$ has indegree $d^{-}(x)$ smaller or equal to $\lambda(x)$ if and only if, for every subgraph $H$ of $G$,

$$
|E(H)| \leq \sum_{x \in V(H)} \lambda(x)
$$

Proof. Actually, (1) translates to the Hoffman condition of the max-flow problem trivially associated to the orientation computation. Nonetheless, let us give a direct short proof of the lemma.

* This work was partially supported by the ESPRIT Basic Research Action Nr. 7141 (ALCOM II). 
Let $G$ be a graph and $\lambda$ be a mapping from $V(G)$ to $\mathbb{N}$. of $G$,

Assume $d^{-}(x) \leq \lambda(x)$, for every vertex $x$ of $G$. Then, for every subgraph $H$

$$
|E(H)| \leq \sum_{x \in V(H)} d^{-}(x) \leq \sum_{x \in V(H)} \lambda(x)
$$

and thus (1) holds.

Conversely, assume (1) holds for any subgraph $H$ of $G$. Consider any orientation of the graph $G$. We will prove the existence of an orientation of $G$ such that $d^{-}(x) \leq \lambda(x)$ for every vertex $x$ of $G$ by induction on the sum

$$
S=\sum_{x, d^{-}(x)>\lambda(x)} d^{-}(x)-\lambda(x)
$$

If $S$ is equal to 0 , the condition $d^{-}(x) \leq \lambda(x)$ holds for every vertex of $G$. Otherwise, let $a$ be a vertex of $G$ such that

$$
d^{-}(a)>\lambda(a)
$$

and let $H_{a}$ be the subgraph of $G$ induced by the vertices $y$ such that there exists a (maybe empty) directed path in $G$ from $y$ to $a$.

If $d^{-}(y) \geq \lambda(y)$, for any vertex $y$ in $H_{a}$, then

$$
\left|E\left(H_{a}\right)\right| \geq \sum_{y \in V\left(H_{a}\right)} d^{-}(y)>\sum_{y \in V\left(H_{a}\right)} \lambda(y)
$$

which contradicts (1). Thus, there exists in $H_{a}$ a vertex $b$ such that

$$
d^{-}(b)<\lambda(b)
$$

By definition of $H_{a}$, there exists in $G$ a directed path from $b$ to $a$. By reversing the orientation of the edges of this directed path, only the indegrees of the vertices $a$ and $b$ are changed and hence the sum $S$ decreases by one.

It is a straightforward consequence of this lemma that a graph $G$ has an orientation such that each vertex has indegree at most $\alpha$ if and only if the average degree of the vertices of any subgraph of $G$ is at most 2. $\alpha$. In particular :

Theorem 2. Let $G$ be a graph and $\kappa(G)$ be the minimum integer such that the graph $G$ has an orientation in which each vertex having at most $\kappa(G)$ incoming edges. Then,

In particular,

$$
\kappa(G)=\max _{H \subseteq G} \frac{|E(H)|}{|V(H)|}
$$

- if $G$ is planar bipartite, $\kappa(G) \leq 2$

- if $G$ is of genus $\gamma(G) \leq 1$, then $\kappa(G) \leq 3$

- if the maximum degree of the vertices of $G$ is $2 . \alpha$, then $\kappa(G) \leq \alpha$

- if $\Upsilon(G)$ denotes the arboricity of $G$, then $\kappa(G) \leq \Upsilon(G)$

- if $p(G)$ denotes the page number of $G$, then $\kappa(G) \leq p(G)+1$ (e.g. $\kappa(G) \leq 2$ if $G$ is outer planar) 


\section{Some Planar Augmentation Problems}

\subsection{Triangulation of triconnected graphs}

Let us give the demonstration of an augmentation problem, where a rather surprising bound arises :

Problem 3. What is the smallest integer $k$, such that any triconnected planar graph can be triangulated by increasing the degrees of the vertices by at most $k$ ?

The resolution of this problem is the consequence of few simple lemmas.

Kant [3] introduced a generalization of the canonical ordering introduced for triangulated planar graphs in [1] :

Lemma (KANT). The vertices of a triconnected graph $G$ can be ordered in a sequence $v_{1}, \ldots, v_{n}$ such that $v_{2}$ and $v_{n}$ are neighbors of $v_{1}$ and are on a common face, and for every $k, k>3$ :

1. $v_{k}$ is on the outerface of $G_{k}$ and has at least two neighbors in $G_{k-1}$, which are on the outerface of $G_{k-1}, v_{k}$ has at least one neighbor in $G-G_{k} . G_{k}$ is biconnected,

2. or there exists an $l \geq 1$ such that $v_{k}, \ldots v_{k+l}$ is a chain on the outerface of $G_{k+l}$ and has exactly two neighbors in $G_{k-1}$, which are on the outerface of $G_{k-1}$. Every vertex $v_{k}, \ldots v_{k+1}$ has at least one neighbor in $G-G_{k+l} . G_{k+1}$ is biconnected.

Remark. Such a canonical ordering can be easily generalized to triconnected non-planar graphs.

Using Kant's Lemma, we deduce :

Lemma 4. Let $G$ be a triconnected planar graph. Then, there exists an orientation of $G$, such that :

- each interior vertex has indegree 2 or 3.

- each face has at most one source.

- this orientation is extendable to a orientation of a triangulation of $G$, each interior vertex having indegree 3.

Proof. We use the canonical ordering defined in Kant's Lemma to perform a triangulation $G_{t}$ of $G$ with the same canonical ordering.

At each step, we add either a vertex $v_{k}$ or a chain $\left\{v_{k}, \ldots v_{k+l}\right\}$. Let $x$ and $y$ be respectively the leftmost and the rightmost vertices of $G_{k-1}$ incident to one of the added vertices. Let some dummy edges be added incident to $v_{k}$ and all the vertices of $G_{k-1}$ between $x$ and $y$ (if not already adjacent to $v_{k}$ ). When a chain is added, we also add dummy edges from $v_{k+1}, \ldots, v_{k+l}$ to $y$.

The canonical order of $G$ is obviously a vertex packing order of the obtained triangulated graph $G_{t}$. 
To an orientation, we associate a marking $\mu$, which maps the angles of the planar map into the integers. The angle $a$ defined by a vertex $x$ and face $f$ has a marking $\mu(a)$ equal to the number of edges of $f$ oriented toward $x$.

If a graph $G$ is a partial graph of a graph $H$, then the angle $a$ of $G$ defined by a vertex $x$ and face $f$ has, by extension, a marking $\mu(a)$ equal to the number of edges of $H$ "inside" $f$ and oriented toward $x$.

Lemma 5. Let $G$ be a triconnected planar graph. Then, there exists a marking $\mu$ such that:

- the sum of the marks of the angles incident to a vertex is smaller or equal to 6 ,

- the sum of the marks of the angles incident to a face of length $l$ is equal to $3 l-6$,

- the mark of any angle a statisfies $0 \leq \mu(a) \leq 3$,

- for any face $\phi$, at most one angle of $\phi$ has mark 0 .

Proof. Consider the orientation of $G$ and the oriented triangulation $G_{t}$ defined by Theorem 4 . The marking defined by $G_{t}$ on the angle of $G$ have the required properties.

The final lemma proves the utility of the so-defined marking :

Lemma 6. Let $\gamma$ be a cycle of length $l$ and let $\mu$ be a marking of the interior angles of $\gamma$, such that:

- the sum of the marks on $\gamma$ is greater or equal to $3 l-6$,

- the marks are smaller or equal to 3 ,

- there is at most one angle of $\gamma$ with mark 0.

Then, there exists a triangulation of the interior of $\gamma$, such that the degree increase of the vertices of $\gamma$ are bounded by the corresponding marks.

Proof. The proof is by induction and case analysis. In the following, the mark of the vertices of $\gamma$ are defined as the mark of the corresponding angle of $\gamma$.

- There exists a vertex $v$ with mark 0 .

Let $x$ and $y$ be the neighbors of $v$. As the marks are smaller or equal to 3 , $\mu(x)+\mu(y) \geq(3 l-6)-3(l-3) \geq 3$. As only one vertex may have a mark equal to 0 , one vertex among $x$ and $y$ has a mark greater or equal to 2 . When adding an edge joining $x$ and $y$ and decreasing by one the marks of $x$ and $y$, we get a cycle of length $l-1$ with the required properties.

- There exists no vertex having a mark equal to 0 , but there exists a vertex having a mark equal to 1 .

A simple calculation shows that there exist at most three vertices of $\gamma$ having a mark equal to 1 . If $l$ is not of length 3 , there exists vertex $v$ having a mark equal to 1 and adjacent to a vertex $x$ having a mark greater or equal to 2 . Let $y$ be the other neighbor of $v$ in $\gamma$. When adding an edge joining $x$ and $y$ and decreasing by one the marks of $x$ and $y$, we get a cycle of length $l-1$ with the required properties. 
- All the marks are greater or equal to 2.

The cycle can be triangulated using a "zig-zag".

Theorem 7. Let $G$ be a triconnected planar graph. Then there exists a triangulation of $G$, such that the degrees of the vertices of $G$ increase at most by 6 . Moreover, this bound is tight.

Proof. The existence of such a triangulation is a direct consequence of Lemmas 5 and 6 . The tightness of the bound is shown by replacing each face of a big maximal planar graph by a prism (see Fig. 3.1).
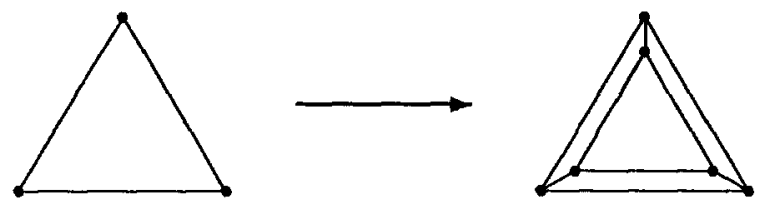

Fig. 1. Construction of a worst-case example

\subsection{Other Triangulation Problems}

The bound obtained above for triangulating triconnected planar graphs may be improved when restricted to special classes of triconnected graphs. Without demonstration, let us mention the results obtained for outerplanar graphs and maximal bipartite planar graphs.

Theorem 8. Let $G$ be a triconnected outerplanar graph. Then there exists a trangulation of $G$, such that the degrees of the vertices of $G$ increase at most by 3.

Theorem 9. Let $G$ be a triconnected maximal bipartite planar graph. Then there exists a triangulation of $G$, such that the degrees of the vertices of $G$ increase at most by 4. Moreover, this bound is tight.

The tightness of the bound is shown by replacing each face of a big maximal bipartite planar graph as shown by Fig. 3.2.

It seems that this bound also holds for any triconnected graph without separating triangle :

Conjecture 10. Let $G$ be a triconnected planar graph free of separating triangles, it is possible to triangulate $G$ by adding at most 4 edges at each vertex. 


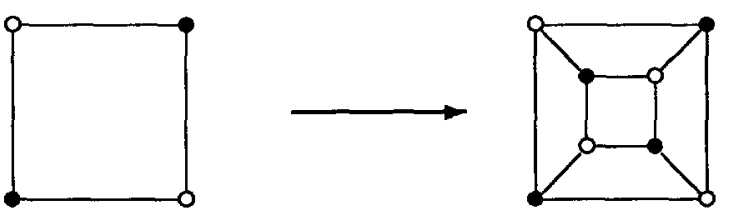

Fig. 2. Construction of a worst-case example

\subsection{Augmentation of connected graphs}

Let us present two other augmentation problems, that have been solved using regular orientations. We shall only give short hints on the way regular orientations are actually used in solving them.

By augmenting $G$ into a maximal planar graph and computing the marking induced by an orientation with indegrees bounded by 3 , we prove :

Theorem 11. Let $G$ be a connected plane graph. Then, $G$ can be augmented to a biconnected plane graph (while preserving the embedding) by increasing the degrees of the vertices of $G$ increase at most by 6 . Moreover, this bound is tight.

The tightness of the bound is shown by replacing each face of a big maximal planar graph the way shown in Fig. 3.3. In the worst-case example given above,
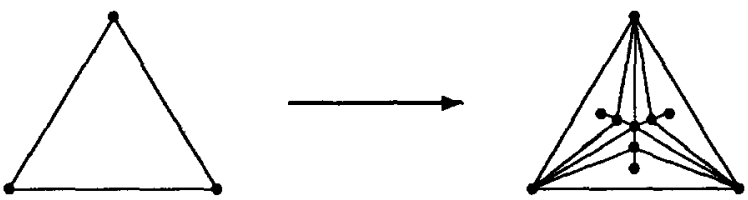

Fig. 3. Construction of a worst-case example

the bound can be improved if the embedding may be changed. Nonetheless, it appears that the choice of the embedding is harder than simply gathering blocs articulated at a same vertex in a same face.

Actually, by using an orientation of the angle graph of a planar graph with indegrees bounded by two, we prove:

Theorem 12. Let $G$ be a connected planar graph. Then, $G$ can be augmented to a biconnected planar graph by increasing the degrees of the vertices of $G$ increase at most by 2. Moreover, this bound is tight.

\section{Remark}

It is straightforward to devise linear time algorithms to solve the augmentation problems presented in this section. 


\section{On tree decompositions}

The arboricity $\Upsilon(G)$ of a graph has been expressed by a formula similar to that for $\kappa(G)[4]$ :

Theorem (Nash-Williams). The arboricity $\Upsilon(G)$ of a graph $G$ is equal to :

$$
r(G)=\max _{H \subseteq G,|V(H)|>1} \frac{|E(H)|}{|V(H)|-1}
$$

It follows that :

- any maximal bipartite planar graph is covered by two trees [8] [6],

- any maximal planar graph is covered by three trees [9] [7].

Actually, a necessary and sufficient condition for a graph $G$ to be the union of $k$ spanning trees may be expressed in terms of regular orientations :

Theorem 13. A graph $G$ with $k(|V(G)|-1)$ edges is the disjoint union of $k$ disjoint spanning trees if and only if there exists an orientation $O$ and a vertex $x_{0}$ of $G$, such that, for any bipartition $V_{0} \cup V_{1}$ of $V(G)$ with $x_{0} \in V_{1}$, the number of edges directed from a vertex of $V_{0}$ to a vertex of $V_{1}$ is at least equal to $k$.

Any tree decomposition canonically induces such an orientation. The converse seems also to be true :

Conjecture 14. Let $k>1$ be an integer and $x_{0}$ be a vertex of an oriented graph $G$ having exactly $k(|V(G)|-1)$ edges.

Then, the following two properties are equivalent:

1. For any bipartition $\left(V_{0}, V_{1}\right)$ of the vertex set of $G$ (with $x_{0} \in V_{0}$ ), there exists at least $k$ distinct edges of $G$ oriented from a vertex belonging to $V_{0}$ to a vertex belonging to $V_{1}$.

2. The graph $G$ is covered by $k$ edge-disjoint trees, such that each vertex (except $x_{0}$ ) has exactly one incoming edge belonging to each tree.

For some classes of planar graphs, tree decompositions may be easily deduced from such orientations. Thus, Schnyder's theorem on the decomposition of a maximal planar graph in to 3 trees may be extended to [5] :

Theorem 15. The Schnyder decompositions of a maximal plane graph into 3 trees are in bijection with orentations such that each vertex not belonging to the unbounded face has indegree 3. Moreover those omentations are compatible with the orientation of the 3 trees oriented from their roots.

For bipartite planar graphs, by slightly different techniques, we prove that :

Theorem 16. The decompostions of a maximal bipartite plane graph into 2 trees rooted in two vertices of the unbounded face, such that the edges belonging to a same tree are consecutive at each vertex, are in bijection with orientations such that each vertex not belonging to the unbounded face has indegree 2. Moreover those orientations are compatible with the orientation of the 2 trees oriented from their roots. 
From this theorem, it follows that any maximal bipartite graphs may be drawn on two pages, with one tree on each page. It seems that such a representation can be generalized to any planar graph covered by two trees. As the page number of any planar graph is at most 3 , this would imply the following inequality :

Conjecture 17. Let $G$ be a planar graph. Then, the page number $p(G)$ of $G$ is related to its arboricity $\Upsilon(G)$ by :

$$
p(G) \leq \Upsilon(G)
$$

\section{References}

1. H. de Fraysseix, J. Pach, and R. Pollack. Small sets supporting Fary embeddings of planar graphs. In Twentieth Annual ACM Symposium on Theory of Computing, pages 426-433, 1988.

2. K.P. Eswaran and R.E. Tarjan. Augmentation problems. SIAM J. Comput., 5:653665, 1976.

3. G. Kant. Algorithms for Drawing Planar Graphs. Ph.D. thesis, Utrecht University, Utrecht, 1993.

4. C. St J. A. Nash-Williams. Edge-disjoint spanning trees of finite graphs. J. London Math. Soc., 36:445-450, 1961.

5. P. O. de Mendez. Orientations bipolaires. Ph.D. thesis, Ecole des Hautes Etudes en Sciences Sociales, Paris, 1994.

6. V. Petrović. Decomposition of some planar graphs into trees. In J. Bolyai Mathematical Society, editor, Proc. International Conference on Combinatorics, page 48, 1993.

7. V. Petrović, G. Ringel, and C. Thomassen. Decomposition of maximal graphs into trees. preprint, 1993.

8. G. Ringle. Two trees in maximal planar bipartite graphs. Journal of Graph Theory, 17(6):755-758, 1993.

9. W. Schnyder. Planar graphs and poset dimension. Order, 5:323-343, 1989.

10. W. Schnyder. Embedding planar graphs in the grid. In First ACM-SIAM Symposium on Discrete Algorithms, pages 138-147, 1990. 